\title{
THEORETICAL STUDY OF SUBSTITUTION OF Si FOR Ge IN $\mathrm{Mn}_{5} \mathrm{Ge}_{3}$
}

\author{
B. Siberchicot, R. Henrion \\ CEA/CEL-V, 94195 Villeneuve-St-Georges, CEDEX, France \\ AND J. TOBOLA \\ Faculty of Physics and Nuclear Techniques, AGH \\ Al. Mickiewicza 30, 30-059 Kraków, Poland
}

\begin{abstract}
The substitution of silicon for germanium in $\mathrm{Mn}_{5} \mathrm{Ge}_{3}$ leads to a decrease in the magnetization for $x<0.7$ and then to phase transition toward a non-collinear antiferromagnetic order for $\mathrm{Mn}_{5} \mathrm{Si}_{3}$. The magnetic properties of $\mathrm{Mn}_{5} \mathrm{Ge}_{3}$ and $\mathrm{Mn}_{5}\left(\mathrm{Ge}_{0.5} \mathrm{Si}_{0.5}\right)_{3}$ are analyzed by means of band structure calculations.
\end{abstract}

PACS numbers: 71.20.Be, 75.10,Lp, 75.50.Cc

\section{Introduction}

The intermetallic compounds of manganese present a lot of crystallographic and magnetic structures. The ternary compounds $\mathrm{Mn}_{5}\left(\mathrm{Ge}_{1-x} \mathrm{Si}_{x}\right)_{3}$ exhibit a complex and rich magnetic phase diagram. Thus this system has been experimentally studied in order to characterize the nature of chemical bondings and magnetic interactions between manganese and metalloid atoms [1]. $\mathrm{Mn}_{5} \mathrm{Ge}_{3}$ is a two sublattices ferromagnet and $\mathrm{Mn}_{5} \mathrm{Si}_{3}$ - a complex non-collinear antiferromagnetic material. Both the compounds adopt hexagonal packing with space group $P 6_{3} / \mathrm{mcm}$. We have undertaken band structure calculations of this system with the use of augmented spherical waves (ASW) [2] and Korringa-Kohn-Rostoker (KKR) [3] methods. The disordered systems were described by KKR method with coherent potential approximation (CPA).

\section{2. $\mathrm{Mn}_{5} \mathrm{Ge}_{3}$}

In the framework of classical theory of binary alloys, a mean value of magnetization could be calculated [4]. For an $\mathrm{A}_{x} \mathrm{~B}_{1-x} \mathrm{Mn}_{0.625} \mathrm{Ge}_{0.375}$ alloy this model leads to the following expression: $\mu_{\mathrm{A}}=\mu_{\mathrm{A}}{ }^{0}-Z_{\mathrm{B}} \frac{x}{1-x}$, where $Z_{\mathrm{B}}$ is the number of valence electrons for the $\mathrm{B}$ metal and $\mu_{\mathrm{A}}^{0}$ - the moment of the magnetic atom. If we assume $\mathrm{Mn}^{2+}$ ions in a high spin state $\left(\mu_{\mathrm{Mn}^{2+}}^{0}=5 \mu_{\mathrm{B}}\right)$ and $Z_{\mathrm{B}}=4$, we obtain a value $\mu_{\mathrm{Mn}}=2.6 \mu_{\mathrm{B}}$ in agreement with experiment. In fact, this model is too 
crude as the manganese atoms are not differentiated. The cell contains two crystallographic independent sets of manganese atoms: $\mathrm{Mn}(4 d)$ in a fourfold position and $\operatorname{Mn}(6 g)$ in sixfold position. The difference in the neighborhood could lead to very different magnetic moments on these two kinds of atoms. Some contradictory models have been proposed to explain these phenomena $[5,6]$, therefore we have undertaken band structure calculations including complementary ASW and KKR methods in order to differentiate the various models. We do not observe significant differences between electronic spectra obtained from ASW and KKR methods, as the spin-polarized density of states (DOS) from ASW method are only presented in Fig. 1. The metallic character of the compound is confirmed even though the DOS

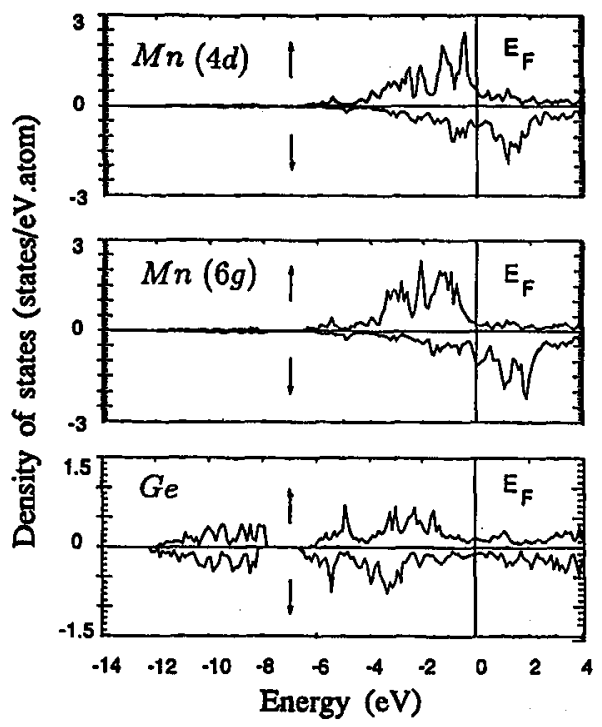

Fig. 1. The spin-projected densities of states of $\mathrm{Mn}_{5} \mathrm{Ge}_{3}$ (from ASW method).

at the Fermi level $\left(E_{\mathrm{F}}\right)$ are weak. The Ge $4 s$ bands are located between -12 and $-8 \mathrm{eV}$ below $E_{\mathrm{F}}$. Beyond $-7 \mathrm{eV}, \mathrm{Ge} 4 p$ and manganese $(\mathrm{Mn}(4 d)$ and $\mathrm{Mn}(6 g)) 3 d$ bands are observed. These bands, crossed by $E_{F}$, are strongly mixed with Ge $4 p$ states leading to a broadening of manganese bands and a slight polarization of Ge which is antiparallel to Mn one. The magnetic moments, hyperfine fields and spin-orbit coupling constants are reported in Table I together with the most recent experimental results [7]. The two calculations give similar results and are in good agreement with these neutron measurements. The two sets of manganese atoms have very different magnetic behavior (various $\mu, H_{\mathrm{hf}}, \zeta_{3 d}$ ), therefore these calculations definitely rule out the previous model by Kanematsu [5] and confirm the measurements of Forsyth and Brown [7].

$$
\text { 3. } \mathbf{M n}_{5}\left(\mathrm{Ge}_{1-x} \mathrm{Si}_{x}\right)_{3}
$$

The effect of the interaction manganese-metalloid may be studied by the substitution of silicon for germanium in the ferromagnetic domain. In a first step 
TABLE I

Calculated magnetic properties of $\mathrm{Mn}_{5} \mathrm{Ge}_{3}$.

\begin{tabular}{c|c|c|c|c|c|c|c|c}
\hline \hline & \multicolumn{9}{|c|}{ ASW } & \multicolumn{2}{|c|}{ KKR } & Exp. \\
\cline { 2 - 8 } & $\begin{array}{c}\mu_{\text {spin }} \\
{\left[\mu_{\mathrm{B}}\right]}\end{array}$ & $\begin{array}{c}\mu_{\text {orb }} \\
{\left[\mu_{\mathrm{B}}\right]}\end{array}$ & $\begin{array}{c}\mu_{\text {tot }} \\
{\left[\mu_{\mathrm{B}}\right]}\end{array}$ & $g$ & $\begin{array}{c}\zeta_{3 d} \\
{\left[\mathrm{~cm}^{-1}\right]}\end{array}$ & $\begin{array}{c}\mu_{\text {tot }} \\
{\left[\mu_{\mathrm{B}}\right]}\end{array}$ & $\begin{array}{c}H_{\mathrm{hf}} \\
{[\mathrm{kGs}]}\end{array}$ & $\begin{array}{c}\mu_{\text {tot }} \\
{\left[\mu_{\mathrm{B}}\right]}\end{array}$ \\
\hline $\mathrm{Mn}(4 d)$ & 2.005 & 0.043 & 2.048 & 2.043 & 436 & 1.87 & 130 & 1.96 \\
$\mathrm{Mn}(6 g)$ & 3.046 & 0.036 & 3.018 & 2.023 & 318 & 3.06 & 260 & 3.23 \\
$\mathrm{Ge}(6 g)$ & -0.060 & -0.007 & -0.067 & 2.556 & - & -0.11 & - & - \\
total & 25.962 & 0.346 & 26.31 & 2.027 & - & 25.3 & - & 27.22
\end{tabular}

TABLE II

Calculated magnetic properties of $\mathrm{Mn}_{5}\left(\mathrm{Ge}_{0.5} \mathrm{Si}_{0.5}\right)_{3}$.

\begin{tabular}{c|c|c|c|c|c|c}
\hline \hline & \multicolumn{5}{|c|}{ ASW } & KKR-CPA \\
\cline { 2 - 7 } & $\begin{array}{c}\mu_{\text {spin }} \\
{\left[\mu_{\mathrm{B}}\right]}\end{array}$ & $\begin{array}{c}\mu_{\text {orb }} \\
{\left[\mu_{\mathrm{B}}\right]}\end{array}$ & $\begin{array}{c}\mu_{\text {tot }} \\
{\left[\mu_{\mathrm{B}}\right]}\end{array}$ & $g$ & $\begin{array}{c}\zeta_{3 d} \\
{\left[\mathrm{~cm}^{-1}\right]}\end{array}$ & $\begin{array}{c}\mu_{\text {tot }} \\
{\left[\mu_{\mathrm{B}}\right]}\end{array}$ \\
\hline $\mathrm{Mn}(4 d)$ & 1.742 & 0.003 & 1.747 & 2.003 & 468 & 1.81 \\
$\mathrm{Mn}(6 g)$ & 2.920 & 0.001 & 2.921 & 2.001 & 392 & 2.97 \\
$\mathrm{Ge}$ & 0.030 & 0.002 & 0.032 & 1.872 & - & -0.13 \\
$\mathrm{Si}$ & 0.073 & 0.000 & 0.073 & 2.014 & - & -0.15 \\
total & 24.178 & 0.024 & 24.202 & 1.980 & - & 24.6
\end{tabular}

$\mathrm{Mn}_{5}\left(\mathrm{Ge}_{0.5} \mathrm{Si}_{0.5}\right)_{3}$ is computed (ASW and KKR-CPA), the magnetic moments are given in Table II. In the case of KKR-CPA calculations, the magnetic moments on metalloid atoms show antiparallel polarization as in $\mathrm{Mn}_{5} \mathrm{Ge}_{3}$ while for ASW the values are slightly positive. In fact, KKR-CPA results are probably more accurate because ASW method is based on the atomic sphere approximation (ASA) and the local magnetic moments (and charges) depend on the choice of sphere radii. The total magnetization is reduced by about $1 \mu_{\mathrm{B}}$. The variation of magnetic moments on manganese atoms seems slightly more important for $\mathrm{Mn}(4 d)$ than for $\mathrm{Mn}(6 g)$. The effect of decrease in the magnetic moments when moving on magnetic diagram from $x=0$ to $x=0.7$ (upper limit of ferromagnetic domain) could be connected with lattice parameters changes and $\mathrm{Mn}-\mathrm{Mn}$ distances. A quasi-linear decrease in cell parameter [8] is observed with increasing silicon content. Experimentally, an empirical law has been written $\mu=(2.48-0.67 x) \mu_{\mathrm{B}}$ which is coherent with our results. For $x>0.7$ the positive exchange contribution between manganese atoms decreases quickly and the total magnetization falls down to zero with $x=0.85$ [9]. Such behavior, at magnetic phase transition region, could be analyzed with further total energy KKR-CPA computations. 


\section{Conclusions}

The difference of coordination of manganese atoms with germanium (and/or silicon) could not completely explain the large difference of magnetic moments between two kinds of $\mathrm{Mn}$. In fact, $\mathrm{Mn}(6 \mathrm{~g})$ atoms have got ten neighbors for eight in the case of $\mathrm{Mn}(4 d)$. In this case two of these neighbors are very close and this suggests a high interaction $\mathrm{Mn}-\mathrm{Mn}$ and then a low magnetic moment. Such a phenomenon has already been observed in a lot of manganese alloys. A better interpretation of chemical bondings $\mathrm{Mn}-\mathrm{Mn}$ and $\mathrm{Mn}-\mathrm{Ge}(\mathrm{Mn}-\mathrm{Si}$ ) could be made by the calculations of magnetic crystal orbital overlap populations (COOP) [10]. Such calculations are now in progress. In another step, the complex antiferromagnetic structure of $\mathrm{Mn}_{5} \mathrm{Si}_{3}$ could be computed using the non-collinear version of ASW code [11].

\section{References}

[1] P. Panissod, A. Qachaou, G. Kappel, in: Proc. Int. Magnetism Conf., Kyoto, North-Holland, Amsterdam 1982, p. 701.

[2] A.R. Williams, J. Kübler, C.D. Gelatt Jr., Phys. Rev. B 19, 6094 (1979).

[3] A. Bansil, S. Kaprzyk, J. Toboła, in: Applications of Multiple Scattering Theory to Materials Science, Eds. W.H. Butler, P.H. Dederichs, A. Gonis, R.L. Weawer, Symposium Proc., Vol. 253, Materials Research Society, Pittsburgh 1992, p. 505.

[4] A.R. Williams, A.P. Malozemoff, V.L. Moruzzi, Phys. Rev. B 29, 1620 (1984).

[5] K. Kanematsu, J. Phys. Soc. Jap. 17, 85 (1962).

[6] J.B. Forsyth, P.J. Brown, in: Proc. Int. Magnetism Conf., Nottingham, The Physical Society, London 1964, p. 524.

[7] J.B. Forsyth, P.J. Brown, J. Phys., Condens. Matter 2, 2713 (1990).

[8] Van Con Kieu, Compt. Rend. Acad. Sci. France 260, 111 (1965).

[9] M.E. Sheinker, R.P. Krentsis, P.V. Gel'd, Sov. Phys.-Solid State 19, 1109 (1977).

[10] R. Hoffmann, Angew. Chem. Int. Ed. Engl. 26, 846 (1987).

[11] M. Uhl, B. Siberchicot, J. Phys., Condens. Matter 7, 4277 (1995). 